\title{
DETERMINACIÓN DE LA VENTANA DEL PROCESO PRODUCTIVO EN LA FABRICACIÓN DE BLOQUES H-10 EN OCAÑA NORTE DE SANTANDER Y LA REGIÓN
}

\section{DETERMINATION OF THE WINDOW OF PRODUCTION PROCESS IN THE MANUFACTURE OF BLOCKS H-10 IN OCAÑA NORTE DE SANTANDER AND THE REGION}

\author{
MSc (c). Ricardo Andrés García León a ${ }^{\text {a }}$ MSc. Eder Flórez Solano ${ }^{\text {b }}$ \\ ${ }^{a}$ Universidad Francisco de Paula Santander Ocaña. Facultad de Ingenierías, Grupo de \\ Investigación Ingap. Vía Acolsure, Sede el Algodonal - Ocaña, Norte de Santander, \\ Colombia. Tel.: +57 5690088 Ext. 210. ncardonad@ufpso.edu.co \\ ${ }^{\mathrm{b}}$ Universidad Francisco de Paula Santander Ocaña. Facultad de Ingenierías, Departamento \\ Ingeniería Mecánica. Vía Acolsure, Sede el Algodonal - Ocaña, Norte de Santander, \\ Colombia. enflorezs@ufpso.edu.co
}

Fecha de recepción: 05-02-2016

Fecha de aprobación: 11-05-2016

\begin{abstract}
Resumen: Actualmente en la región Norte de Santander, específicamente en Ocaña, existe solo una empresa que cuenta con la tecnología adecuada en cuanto a tecnificación del proceso productivo de bloques $\mathrm{H}-10$, y debido a su gran capacidad de adsorción este producto es muy solicitado por los constructores de la región y sus alrededores, es por esta razón que surge la iniciativa de estudiar el proceso en cada una de sus etapas con la finalidad de realizar un reconocimiento de las variables actuales que intervienen en el proceso de fabricación del bloque tales como: Procesamiento, proporciones, materiales, componentes, etc. En donde posteriormente se realizaran diferentes estudios tecnológicos a nivel laboratorio con los cuales se podrá optimizar el proceso productivo en lo que concierne a la mezcla optima de material arcilloso, debido a que la etapa de mezcla es considerada como la más crítica. Por consiguiente, con esta investigación se espera aumentar la rentabilidad de la empresa disminuyendo los desperdicios que actualmente superan el $8 \%$ ayudando a una mayor productividad y calidad en las piezas fabricadas, asimismo generando un menor costo en la producción.
\end{abstract}

Palabras clave: Ventana de Proceso, Bloques H-10, Arcilla, Mezcla, Desperdicios.

Abstract: Currently in the northern region of Santander, specifically in Ocana, there is only one company that has the right technology as far as automation of the production process block $\mathrm{H}-10$, and due to its high adsorption capacity this product is in demand by builders of the region and its surroundings is for this reason that the initiative to study the process in each 
of its stages in order to conduct survey of current variables involved in the manufacturing process of the block such as arises: processing, proportions, materials, components, etc. Where further laboratory studies different technological level which can optimize the production process with respect to the optimal mix of clay material, because the mixing step is considered the most critical were made. Therefore, this research is expected to increase profitability by decreasing waste currently helping more than $8 \%$ higher productivity and quality in the manufactured parts also generating lower costs in production.

Keywords: Process window Blocks H10, Clay, Mixing, Waste.

\section{INTRODUCCIÓN}

El proceso cerámico se compone principalmente de tres fases, que son; preparación de la pasta cerámica, moldeo de la pieza y cocción. En la primera fase del proceso se modifica la composición y la plasticidad de la arcilla con el fin de obtener una pasta cerámica homogénea, luego se moldea según la forma deseada, presión o extrusión. Una vez, se ha obtenido la pieza moldeada se procede al secado controlando la velocidad de evaporación de agua con el fin de evitar defectos en la producto. Posteriormente, se somete al proceso de cocción con el fin disminuir la porosidad, aumentar la densidad y la resistencia mecánica (Muñoz Meneses R. A., Muñoz Chaves J.A., Mancilla P, 2007) (Barranzuela, 2014) (Jácome Manzano, 2012).

El proceso productivo es manejado en tres medidas estratégicas: la explotación minera, la transformación de la arcilla y la comercialización del producto final. Una de las etapas definitivas de este proceso es la cocción, realizada en los hornos. Para su ejecución, el horno pasa por tres etapas: precalentamiento, quema y enfriamiento; deben controlarse en forma correcta, con la finalidad de obtener productos con defectos mínimos y reducir el impacto ambiental (García León, Flórez Solano, \& Acosta Pérez, 2015).

De acuerdo a su capacidad de producción y desarrollo tecnológico las industrias ladrilleras se han clasificado en chircales, ladrilleras pequeñas, medianas y grandes (Riojas Castillo \& Rodríguez Montaña, 2004).

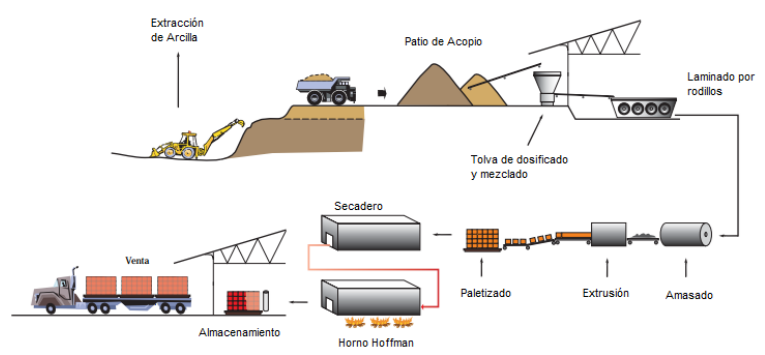

Figura 1. Esquema de fabricación del bloque. Fuente: (Rodríguez, 2007)

En su mayoría las arcillas se destinan a la industria cerámica de la construcción, un 90 $\%$ de la producción se dedica, preferentemente a la fabricación de materiales de construcción y agregados. Sólo un $10 \%$ se dedica a otras industrias (fabricación de papel, caucho, pinturas, absorbentes, decolorantes, arenas de moldeo, productos químicos y farmacéuticos, agricultura, etc.) (Emilia \& Suárez Barrios, 2004).

Estos materiales, son muy usados a nivel de la construcción y otros procesos industriales, pueden ser clasificados atendiendo a multitud de parámetros; así, las minas que contienen minerales arcillosos en su composición pueden ser clasificados dependiendo de su mineralogía, composición química, origen geológico, propiedades físicas, utilización industrial, comportamiento geotécnico, entre otras 
clasificaciones, pero siendo la más común y la más conocida la clasificación mineralógica, entre las que podemos encontrar, según su estructura molecular, arcillas de tipo caolín, illita y montmorillonita (Díaz Rodríguez \& Torrecillas, 2002).

En el área Norte Santandereana existe gran diversidad de productos que se pueden fabricar con esta materia prima, Colombia en estos momentos no es una gran potencia en fabricación industrial de productos a base de arcillas, sin importar que en los suelos exista una gran abundancia de arcillas óptimas. Para el caso de Norte de Santander existe gran variedad de yacimientos naturales; pero a pesar de esto, es un departamento cuya exportación de materiales a base de arcilla no superan el $5,7 \%$ del total de las exportaciones a nivel regional, representando para la economía del departamento aproximadamente 13,7 millones de dólares a Julio de 2013; y teniendo en cuenta que estas cifras, en relación con materiales para la construcción, sólo se ve reflejada para el municipio de Cúcuta y su área metropolitana, pues es el único municipio que posee PYMES en lo referente a fabricación de materiales arcillosos (FEDESARROLLO, 2012) (Montoya rivas \& Montoya rivas, 2014).

Generalmente, se utilizan tres componentes que desempeñan papeles importantes en la optimización del rendimiento de las propiedades finales de los bloques $\mathrm{y}$ materiales cerámicos.

El primer componentes es la arcilla, por lo que su plasticidad facilita la estructura del producto, mientras que el segundo es el feldespato o alúmina $\left(\mathrm{Al}_{2} \mathrm{O}_{3}\right)$ que se utiliza para fundente y la tercera es el sílice $\left(\mathrm{SiO}_{2}\right)$ que se utiliza como un material de relleno y estabilizador (Kamseu et al., 2007) (Monteiro \& Vieira, 2004). Estas composiciones se determinan mediante la composición química, la cual es la base de la clasificación modernas de los minerales y la aproximación de las mezclas con los diagramas ternarios (Junkes, Carvalho, Segad??es, \& Hotza, 2011) (Martinez, 2012) (Lassinantti Gualtieri, Romagnoli, \& Gualtieri, 2011).

\section{METODOLOGÍA}

Como objeto de muestra se tuvo en cuenta la una de las empresas de la región. La cual fue fundada en el año 1999 en donde se construyó el horno Hoffman de tiro inducido mediante un extractor de aire y tiene la capacidad de producir 23500 bloques H-10 diarios, aproximadamente 700.000 bloques cerámicos mensuales, el cual consta de un sistema con 24 recamaras, las cuales poseen una capacidad de hasta 5 apiles por recamara y de 650 ladrillos secos aproximadamente por apiles, con una separación de $60 \mathrm{~cm}$ entre cada apile de ladrillos. Así mismo, la construcción de un secadero natural de $2586 \mathrm{~m}^{2}$ y en el año 2003 se construye un secadero artificial para 6000 piezas diarias. La quema es realizada atreves de carbón mineral, donde cada ladrillo tiene un consumo promedio de 0.288 Kg (Payares Perez, 2014) (Jacome Manzano, 2015).

Entre el año 2010 y 2011, se adquieren dos equipos de mayor capacidad en la línea de producción (Un mezclador y molino laminador 800), en donde deja la posibilidad de desarrollar y mejorar la calidad del producto actual. Además se amplía 18 metros más el horno Hoffman quedando de 72 metros y se constituye un nuevo secadero artificial con tecnología que minimiza el impacto ambiental. 
El 25 de noviembre de 2015, la empresa logra certificarse en los Sistemas Integrados de Gestión (ISO 9001, OHSAS 18001 e ISO 14001) en su proceso de fabricación de productos derivados de la arcilla y productos de mampostería.

A continuación se presenta el proceso que sufre la arcilla para la fabricación de ladrillos en la empresa Ladrillera Ocaña (Gerencia Hora Ltda, 2015).

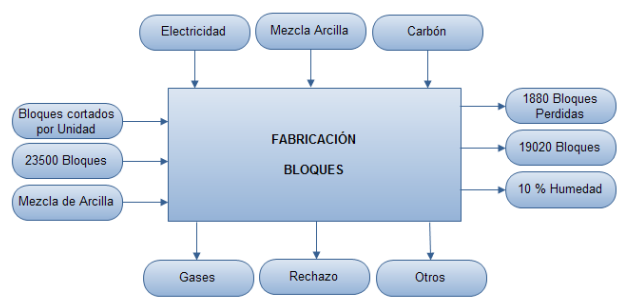

Figura 1. Matriz del proceso.

Fuente. Los Autores

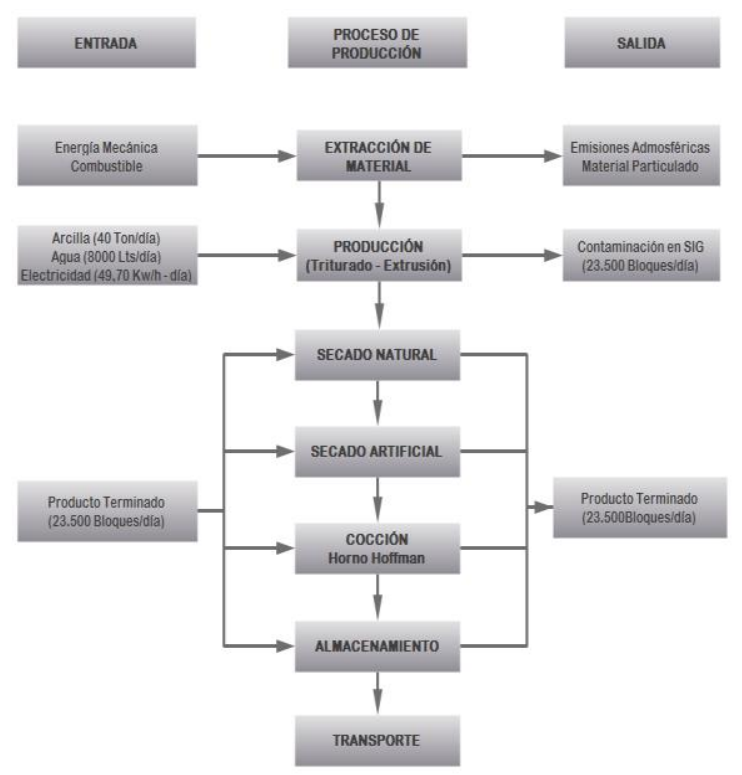

Figura 2. Matriz de Entradas y Salidas

Fuente. Los Autores

Las características morfológicas o dimensionales del bloque H10 son largo, ancho, altura del piso a la pieza, espesor y peso, relacionadas en la Figura 3.

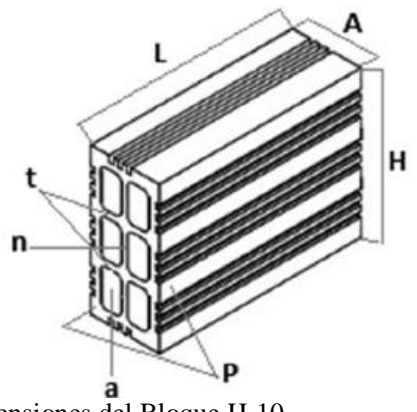

Figura 2. Dimensiones del Bloque H-10

Fuente: (Rozo Rincón, Sánchez Molina, \& Alvarez Rozo, 2014)

Dónde:

$\mathrm{L}=\operatorname{Largo}(\mathrm{cm})$

$\mathrm{A}=$ Ancho $(\mathrm{cm})$

$\mathrm{H}=$ Alto $(\mathrm{cm})$

$\mathrm{P}=$ Espesor más pequeño de las paredes $(\mathrm{mm})$

$\mathrm{T}=$ Espesor más pequeño de los tabiques (mm)

$\mathrm{N}=$ Espesor del nervio ( $\mathrm{mm}$ )

Las dimensiones los bloques H-10 oscilan dependiendo la tecnología utilizada por la empresa y para el caso del bloque de seis huecos que es el más común, los valores de largo varían entre 28,00 y $30,50 \mathrm{~cm}$, y los valores del ancho entre 9,64 y 10,46 cm (Rozo Rincón et al., 2014).

\section{RESULTADOS Y DISCUSIÓN}

Mediante una retroexcavadora, se trasporta la materia prima de la mina existente en los patios de la empresa hacia la tolva de alimentación, estas arcilla utilizadas actualmente son referenciadas como pilas de arcillas verdes y rojas en donde se controla que los porcentajes de la mezcla no sobrepasen el $40 \%$ de arenas, en esta etapa se da inicio al proceso de reducción de la materia prima que generalmente se agrupa en forma de roca (Terrones), para luego ser distribuido en tamaños de partículas menores en las demás etapas del proceso por medio de bandas trasportadoras. 
La materia prima sale aproximadamente con terrones de diámetro entre $10 \quad \mathrm{y} \quad 25$ milímetros.

\section{Transporte de la materia prima}

Para dirigir el material en todo el proceso productivo hasta el final, se utilizan bandas transportadoras guiadas por rodillos, estas bandas son un equipo adecuado para el transporte de arcillas para ser transformados en materiales para la mampostería en este caso Bloques H-10. Según lo informado por la persona encargada de producción y el laboratorio, se alcanza una producción entre 9 a 11 toneladas hora, este mecanismo se caracteriza por el fácil mantenimiento de su estructura.

\section{Trituración del material "Destronador".}

El primer proceso que recibe la materia prima es realizado por el desterronador , donde da inicio a la etapa de trituración de la arcilla, se lleva a cabo mediante las cuchillas montadas sobre los dos ejes robustos paralelos que giran a diferentes velocidades, gracias al movimiento giratorio de las cuchillas están se incrustan sobre el material y producen desgarre del mismo y reduciendo el tamaño del material entre aproximadamente 0.1 y 50 milímetros siendo la dimensión adecuada para seguir el proceso en el desintegrador.

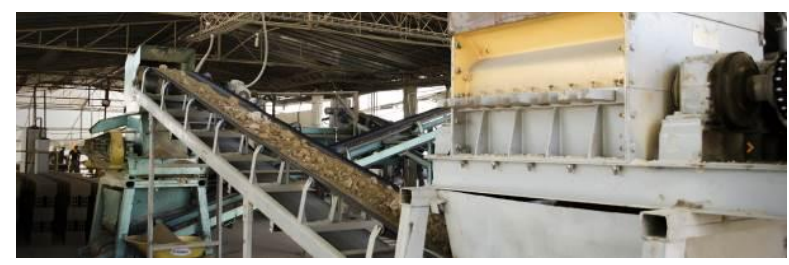

Figura 4: Trituración y transporte Fuente: Los Autores

Disminución del tamaño del grano de la arcilla en el Desintegrador.
Luego de pasar el material por el desterronador, este se transporta por bandas al desintegrador, este equipo adecuado para la trituración de terrones de arcilla, el cual los transforma en granulometrías de entre 0.5 y 20 milímetros. Este equipo se caracteriza por la trituración uniforme que realiza al material y facilita el trabajo de las siguientes maquinas.

\section{Laminado de la arcilla}

Seguidamente trasportada la materia prima por banda del desintegrador esta se traslada al Molino Laminador I, el cual es de alta resistencia al desgaste y de molienda fina, gracias a su compacta estructura, es más estable en funcionamiento, poco ruidoso y con menos polvo en el proceso. Esta máquina es imprescindible para la molienda de arcilla, además es la adecuada para la reducción del material, debido a que produce una masa uniforme que garantiza una mayor calidad del moldeo.

\section{Amasado del material "Amasadora I".}

El material anteriormente era transportando por banda para seguir con el proceso de amasado en la Amasadora I, este mezclador es un equipo adecuado para la homogenización y mezclado de la arcilla. En esta etapa del proceso se le adiciona la cantidad de agua requerida si es necesario dependiendo el material procesado. Actualmente, debido a que realizó un análisis en los costos en el consumo de energía, el resultado arrojó que el equipo podía ser desactivado sin implicar defectos en el proceso y en la calidad del producto y de esta manera reducir los costos.

\section{Paso del material por el Laminador II.}

Luego de salir la materia prima del Molino Laminador I, se trasporta por banda al Laminador II, en donde se realiza el mismo proceso del molino laminador y transporta la 
mezcla de una forma adecuada a la Amasadora II. En esta etapa del proceso la arcilla llega con un porcentaje de humedad de aproximadamente el $16 \%$ en donde se le adiciona agua continua al paso de la banda transportadora en un 3\%, es decir que sale con un $19 \%$ de humedad a la siguiente etapa.

\section{Paso del material por la Amasadora II.}

Al paso de la arcilla por el Molino Laminador II, al igual que en todos los procesos este también se traslada por bandas hacia la Amasadora II, que realiza la misma función de la primera pero las paletas mezcladoras son menos compactas y más pequeñas, en este proceso la mezcla de arcilla es trabajada para pasar finalmente a la extrusora, el tamaño de las partículas de arcilla es menor de 2 milímetros y no se le adiciona más agua, pero en algunas ocasiones dependiendo de la conformación de las arcillas es necesario agregar un poco más de agua con la manguera dispuesta en la máquina.

\section{Extrusión de la arcilla.}

La arcilla después de pasar por todas las etapas anteriores, ya se encuentra con su mezcla adecuada y de esta manera se traslada por bandas a la extrusora, su principal función es extrusión de materiales arcillosos en conformación semihúmeda como seca. En su proceso, la arcilla entra por la parte superior de la maquina donde cae en un sistema de dos ejes paralelos con paletas que reciben la mezcla y la van llevando hacia el interior del tornillo sin fin que se encarga de impulsar la mezcla para la extrusión de la arcilla en un ladrillo compacto. La bomba de vacío, funciona en conjunto con la extrusora de ladrillos, ya que esta presta la función de extraer las moléculas de vapor de agua que se generan en el proceso de extrusión del ladrillo, también ayuda a extraer sustancias no deseadas del producto, asimismo se tiene en cuenta que la bomba no se baje de un vacío de 23 PSI (Vacío y Presión, 2016).

\section{Corte del bloque.}

Se utiliza una cortadora automática, su principal función es realizar el corte del material ya extruido en el tiempo y distancia requerida para la elaboración del bloque $\mathrm{H}$ 10. Tiene un excelente mecanismo que brinda una confiabilidad en sus cortes alcanza una producción de entre 58 y 60 Bloques por minuto alcanzando una producción diaria de 23.500 Bloques por día según la información suministrada por la persona encargada de producción y el laboratorio, luego estos ladrillos finalmente son transportados en carretas y almacenado en los secadores.

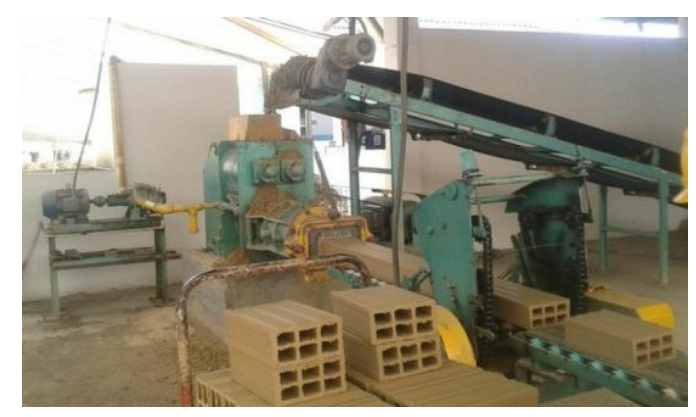

Figura 5. Extrusión y Corte.

Fuente: Los Autores

\section{Secado natural.}

Luego que los bloques son extruidos con aproximadamente un $20 \%$ de humedad son secados a condiciones ambientales durante 5 a 7 días en donde se elimina el agua casi un $8 \%$ de humedad, este proceso ocurre en el patio de secado o ramadas, los cuales son apilados en columnas de 10 bloques y 19 Bloques dependiendo del espacio y son acumulados 4.480 Bloques y los restantes 19.020 a secado artificial, para después ser transportados al secadero artificial con la finalidad de no generar deformaciones $\mathbf{o}$ 
transformaciones bruscas en el material antes de pasar al secado artificial donde las temperaturas son mayores. (Morales Galoc, 2012) (Ver Figura 6).

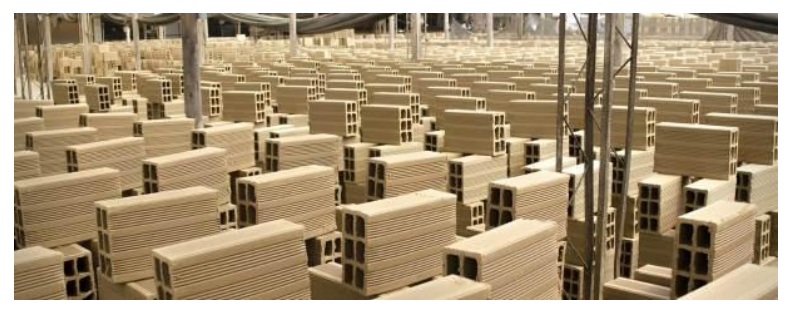

Figura 6. Patio de secado natural Fuente: Los Autores

\section{Conducción de aire caliente a secaderos.}

Para el secado adecuado de los ladrillos que van hacer quemados en el horno es necesario crear un ambiente a alta temperatura para disminuir el porcentaje de humedad, esto de realiza atreves de un extractor, que es un equipo que se encarga de inyectar o conducir el calor que produce la secado artificial.

\section{Secaderos Artificial}

Para la recirculación del aire caliente brindado por el extractor se utiliza un carro con ventilador de recirculación, este carro de translación es un equipo adecuado para agitar y homogenizar el flujo de aire caliente de $50^{\circ} \mathrm{C}$ dentro de la cámara de secado de manera que facilite que el calor se sature de la humedad contenida en el material que se está secando, que son aproximadamente 19.020 bloques/día, este proceso se realiza en la jornada nocturna en aproximadamente 16 horas y en el día es descargado y cargado nuevamente. En esta etapa los bloques pierden entre 5 y $8 \%$ de la humedad.

\section{Preparación del ladrillo de Quema en el Horno Hoffman}

Consiste en 2 galerías paralelas, formadas por compartimientos contiguos, en cuyos extremos se unen por un pasa-fuegos. Son hornos continuos de alta producción, donde no se puede producir materiales vitrificados. En esta etapa el fuego se mueve a través del horno en dirección opuesta a las manecillas del reloj, este sistema permite obtener una alta eficiencia térmica y de producción, ya que el calor obtenido en la cámara de combustión se utiliza en el precalentamiento de las cámaras precedentes.

Aproximadamente una semana es el tiempo que toma realizar un ciclo completo de quema (El fuego llega al punto de donde partió). Cada galería está formada por varias cámaras, cada una de ella con su respectiva puerta en donde se almacena en promedio entre 2500 y 3000 bloques por arrumes de entre 620 y 650 bloques, para el cargue y descargue del horno y un canal de salida que va al colector principal que conduce a la chimenea, cada cámara comunica con el colector, por un conducto de humos, los cuales se cierran herméticamente con válvulas. Para mantener la circulación horizontal de los gases a través de las cámaras. La alimentación del combustible se realiza en la parte superior del horno, mediante alimentación manual o con la ayuda de Carbojet (alimentación neumática), la cual debe realizarse en forma dispersa, evitando chorros que provoquen combustión incompleta.

Esta empresa está dedicada a la producción en serie de ladrillos para la construcción, cuentan con un horno tipo Hoffman, que está compuesto de 24 cámaras repartidas en 12 por cada costado, para la quema continua de ladrillo $\mathrm{H}-10 * 30$ y $\mathrm{H}-10 * 40$ principalmente repartidos en apiles por todo el horno, el combustible utilizado para la cocción del material es el carbón mineral, donde el consumo de carbón por mes es de 170 Ton/mes. 


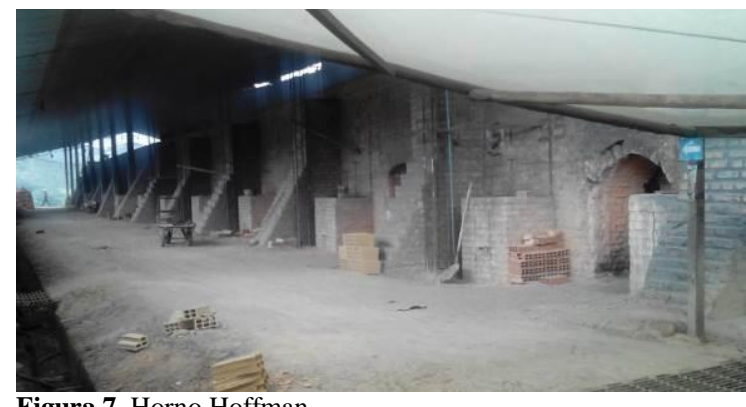

Figura 7. Horno Hoffman.

Fuente: (Jacome Manzano, 2015)

\section{Inyección del carbón al horno de quemado de bloques.}

Este proceso se realiza con un equipo llamado carbojet, según lo informado este equipo es adecuado para la inyección del carbón al proceso de cocción de los materiales, alcanza una inyección de carbón de $8 \mathrm{~kg}$ a $12 \mathrm{~kg}$ por minuto para mantener la temperatura requerida en el horno.

Los bloques al iniciar este proceso con 6 o 7 $\%$ de humedad son trasladados en carros y apilados dentro de las cámaras del horno, el cual alcanza temperaturas de hasta $1200^{\circ} \mathrm{C}$.

Las pérdidas totales de producción de bloques son aproximadamente del $8 \%$ según la información suministrada por la persona encargada de producción y el laboratorio y de los bloques que más se tiene pérdidas son los $\mathrm{H}-10 * 40$ debido a sus dimensiones.

\section{CONCLUSIONES}

Con el desarrollo de esta investigación, se pudo evidenciar que es muy importante tener un control sobre todas las variables del proceso productivo del bloque $\mathrm{H}-10$ con la finalidad de evitar las pérdidas del producto por deformaciones y defectos.

Por otra parte, se logró definir que la etapa del proceso en la que se debe tener más control es la de mezclado y triturado, debido a que si los porcentajes de arenas, limos, arcillas y demás componentes, inciden directamente en el comportamiento del producto en cada una de las etapas.

Es de vital importancia caracterizar las arcillas químicamente con el objetivo de poder diseñar el producto de acuerdo a la tecnología implementada por la empresa y de esta manera poder optimizar los recursos de la empresa y así los desperdicios generados.

En trabajos futuros, se establecerá un procedimiento en cuanto a la formulación de la mezcla óptima de arcilla la cual contenga los porcentajes apropiados de material cerámico, con la finalidad de controlar el proceso como también optimizar los recursos.

\section{FINANCIACIÓN}

Los autores agradecen a la División de Investigación y Extensión de la Universidad Francisco de Paula Santander Ocaña, por su apoyo en la financiación de traslados y el desarrollo de pruebas experimentales.

\section{BIBLIOGRAFÍA}

Barranzuela, J. (2014). Proceso productivo de los ladrillos de arcilla producidos en la región piura. Universidad de Piura.

Díaz Rodríguez, L. A., \& Torrecillas, R. (2002). Arcillas cerámicas: Una revisión de sus distintos tipos, significados y aplicaciones. Boletin de La Sociedad Espanola de Ceramica Y Vidrio, 41(5), 459-470.

Emilia, M. G. R., \& Suárez Barrios. (2004). 
Las arcillas (propiedades y usos). Universidad Complutense (Madrid); Universidad de Salamanca, 25.

FEDESARROLLO. (2012). La Mineria En Colombia : Impacto Socioeconómico y Fiscal. Psikhologitcheskii Jurnal Revue Psychologique, 17(2), 46-55. Retrieved from

http://www.fedesarrollo.org.co/wpcontent/uploads/2011/08/La-mineriaen-Colombia-Informe-de-Fedesarrollo2008.pdf

García León, R. A., Flórez Solano, E., \& Acosta Pérez, M. A. (2015). Análisis estructural de una máquina prensadora para producción de ladrillo macizo para las pequeñas industrias artesanales de materiales cerámicos en ocaña norte de santander y en la región. Revista Colombiana de Tecnologías de Avanzada, 1(1692-7257), 7.

Gerencia Hora Ltda. (2015). Empresa Hora Ltda - Ladrillera Ocaña. Ocaña, Norte de Santander.

Jacome Manzano, S. A. (2015). Evaluación termodinámica del proceso de cocción y análisis de gases en hornos a cielo abierto y Hoffman en Ocaña. Uniersidad Francisco de Paula Santander Ocaña.

Jácome Manzano, S. A. (2012). Evaluación termodinámica del proceso de cocción y análisis de gases en hornos a cielo abierto y Hoffman en Ocaña (Universida). Ocaña: Repositorio ufpso.

Junkes, J. A., Carvalho, M. A., Segades, A. M., \& Hotza, D. (2011). Ceramic tile formulations from industrial waste. InterCeram: International Ceramic Review, 60(1), 36-41.
Kamseu, E., Leonelli, C., Boccaccini, D. N., Veronesi, P., Miselli, P., Pellacani, G., \& Melo, U. C. (2007). Characterisation of porcelain compositions using two china clays from Cameroon. Ceramics International, 33(5), 851-857. http://doi.org/10.1016/j.ceramint.2006. 01.025

Lassinantti Gualtieri, M., Romagnoli, M., \& Gualtieri, A. F. (2011). Influence of body composition on the technological properties and mineralogy of stoneware: A DOE and mineralogicalmicrostructural study. Journal of the European Ceramic Society, 31(5), 673685.

http://doi.org/10.1016/j.jeurceramsoc.2 010.12.002

Martinez, Y. Q. (2012). Caracterización fisicoquímica de cuarzo en el municipio de la playa de belén (Norte de Santander). Universidad Francisco de Paula Santander Ocaña, 84.

Monteiro, S. N., \& Vieira, C. M. F. (2004). Influence of firing temperature on the ceramic properties of clays from Campos dos Goytacazes, Brazil. Applied Clay Science, 27(3-4), 229234. http://doi.org/10.1016/j.clay.2004.03.0 02

Montoya rivas, G. P., \& Montoya rivas, R. (2014). Caracterización del sector cerámico tradicional del valle de Aburrá y los riesgos profesionales latentes en su proceso productivo. Lámpsakos, 12, 34-42.

Muñoz Meneses R. A., Muñoz Chaves 